\title{
CORRIGENDUM
}

\section{Multiscale preferential sweeping of particles settling in turbulence - CORRIGENDUM}

\author{
Josin Tom and Andrew D. Bragg \\ doi:10.1017/jfm.2019.337, Published by Cambridge University Press, \\ 20 May 2019
}

In Tom \& Bragg (2019) the ordinate axis label of figure 5 was incorrect. The correct version of this figure is shown below.

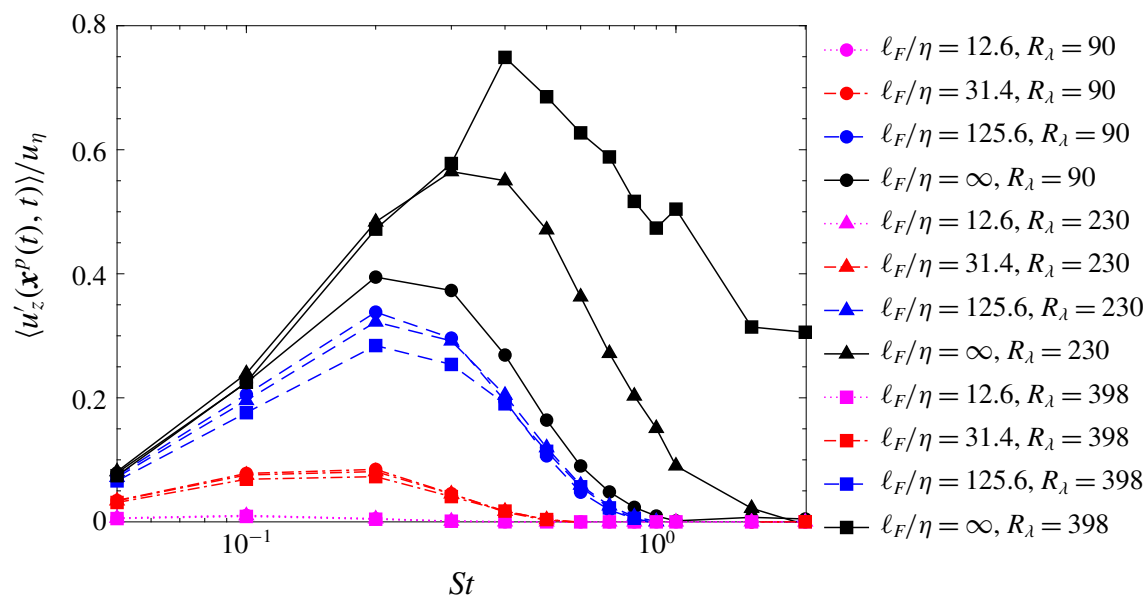

FIGURE 5. (Colour online) DNS results for $\left\langle u_{z}^{\prime}\left(\boldsymbol{x}^{p}(t), t\right)\right\rangle / u_{\eta}$ as a function of $S t$, for $F r=0.052$, and for various filtering lengths $\ell_{F} / \eta$. The circles correspond to $R_{\lambda}=90$, the triangles to $R_{\lambda} \approx 230$ and the squares to $R_{\lambda}=398$. The solid lines correspond to $\ell_{F} / \eta=\infty$, the dashed lines to $\ell_{F} / \eta=125.6$, the dash-dot lines to $\ell_{F} / \eta=31.4$, and the dotted lines to $\ell_{F} / \eta=12.6$.

\section{REFERENCE}

Tom, J. \& BRAGG, A. D. 2019 Multiscale preferential sweeping of particles settling in turbulence.

J. Fluid Mech. 871, 244-270. 